\title{
THE INTERPLAY BETWEEN THE NUCLEAR BARS, CENTRAL STARBURST, AND REMARKABLE OUTFLOW IN NGC 2782
}

\author{
S. JOGEE AND J.D.P. KENNEY \\ Yale University, New Haven, CT 06520-8101, U.S.A. \\ AND \\ B.J. SMITH \\ IPAC/Caltech, Pasedena, CA 91125
}

We show that the nearby peculiar interacting galaxy NGC 2782 (Arp 215) harbors a clumpy molecular $\mathrm{CO}$ bar. The gas bar has a radius of $\sim 7.5^{\prime \prime}(1.3$ $\mathrm{kpc}$ ), a mass of $\sim 2.4 \times 10^{9} M_{\odot}$, and leads a nuclear stellar bar of similar extent (Jogee et al. 1997b). We estimate the gravitational torque exerted by the nuclear stellar bar and find large gas inflow rates $\left(\gg 1 M_{\odot} \mathrm{yr}^{-1}\right.$ ) into the central $200 \mathrm{pc}$ where the starburst activity peaks. We suggest that the nuclear gas bar will disappear on timescales ranging from few $\times 10^{8}$ years to a Gyr, under the effect of star formation, dynamical friction, and gravitational torque. We also show that NGC 2782 harbors a starburstdriven outflow with a remarkable bubble morphology that has not been observed to date in any other starburst galaxy of comparable luminosity (Jogee et al. 1997a). The outflow is driving hot, warm, and possibly cold phases of the ISM out of the central kpc. The estimated outflow rate is less than the inflow rate, and this suggests the dynamical mass in the inner $100 \mathrm{pc}$ might increase by several folds within a Gyr. These results might be relevant to theory (Shlosman et al. 1989) and simulations (Friedli \& Martinet 1993) which propose nuclear bars and 'bars within bars' as efficient mechanisms for fueling central starbursts/AGNs.

\section{References}

Friedli, D., \& Martinet, L. 1993, $A \& A, \mathbf{2 7 7}, 27$

Jogee, S., Kenney, J. D. P. \& Smith B. J., 1997a, $A p J L$, in press.

Jogee, S., Kenney, J. D. P. \& Smith B. J., 1997b, $A p J$, submitted.

Shlosman, I., Frank, J., \& Begelman, M. C. 1989, Nature, 338, 45 